\title{
Linear Optimality Theory as a Model of Gradience in Grammar
}

\author{
Frank Keller \\ School of Informatics, University of Edinburgh \\ 2 Buccleuch Place, Edinburgh EH8 9LW, UK \\ phone: +44-131-650-4407, fax: +44-131-650-6626 \\ email: keller@inf.ed.ac.uk
}

\begin{abstract}
This paper provides an overview of Linear Optimality Theory (LOT), a variant of Optimality Theory (OT) designed for the modeling of gradient acceptability judgment data. We summarize the empirical properties of gradient data that have been reported in the experimental literature, and use them to motivate the design of LOT. We discuss LOT's notions of constraint competition and optimality, as well as a new formulation of ranking argumentation, which makes it possible to apply standard parameter estimation techniques to LOT. Then the LOT model is compared to Standard OT, to Harmonic Grammar, and to recently proposed probabilisitic versions of OT.
\end{abstract}

\section{Introduction}

This paper provides an overview of Linear Optimality Theory (LOT), a variant of optimality theory initially proposed by Keller (2000) to model gradient linguistic data. It is important to note that LOT is a framework designed to account for gradient judgment data; as has been argued elsewhere in this volume (Crocker \& Keller, 2004), gradience in processing data and in corpus data has different properties from gradience in judgment data, and it is unlikely that the two types of gradience can be accounted for in a single, unified framework.

The remainder of the paper is structured as follows. In Section 2, we summarize the empirical properties of gradient judgments that motivate the design of LOT. Section 3 defines the components of an LOT grammar, and introduces the LOT notions of constraint competition and optimality. Based on this, ranking argumentation is defined, an algorithm for computing constraint ranks is introduced, and a measure of model fit in LOT is defined. Finally, Section 4 provides a comparison with other variants of OT, particularly with Standard OT and with Harmonic Grammar. This section also contains a survey of more recent

To appear in Gisbert Fanselow, Caroline Féry, Ralph Vogel, and Matthias Schlesewsky, eds., 
developments, such as Probabilistic OT and variants of OT based on maximum entropy models.

\section{Empirical properties of gradient judgments}

Reviewing experimental data covering a range of syntactic phenomena in several languages, Sorace and Keller (2004) identify a number of sources of gradience in grammar. The two central experimental findings according to Sorace and Keller (2004) are that constraints are ranked and that constraint violations are cumulative. Constraint ranking means that some constraint violations are significantly more unacceptable than others. Cumulativity means the multiple constraint violations are significantly more unacceptable than single violations. These properties seem to be fundamental to the explanation of gradient linguistic judgments and therefore should form the basis of a model of gradience in grammar. Cumulativity also accounts for the ganging up of constraints: multiple violations of lower ranked constraints can be as unacceptable as a single violation of a higher ranked constraint. Experimental results reported by Keller (2000) show that a ganging up effect can be observed for constraints on word order, extraction, and gapping

Another central experimental result identified by Sorace and Keller (2004) is that constraints can be classified into two types, soft and hard. While both types of constraint share the properties of ranking and cumulativity, they differ in another set of properties (as summarized in Table 1). First, soft constraint violations are associated with mild unacceptability, while hard violations trigger serious unacceptability. Second, soft constraints are context-dependent, while hard constraints are immune to context effects. Third, only soft constraints are subject to developmental optionality in first and second language acquisition and in language attrition: hard constraints are developmentally stable, even in second language speakers and in first language speakers subjects to attrition.

A fourth property of gradient structures concerns crosslinguistic variation. Sorace and Keller (2004) argue that both hard and soft constraints are subject to crosslinguistic variation; however, they hypothesizes that crosslinguistic variation cannot affect the type of a constraint, i.e., they predict that there are no constraints that are soft in one language and hard in another. (Note that this presumes that there is an inventory of universal constraints for all languages, as assumed in Optimality Theory.)

Based on these properties, we the notion of constraint type can be can operationalized. If a constraint violation induces strong unacceptability and fails to show context effects and developmental optionality, then it can be classified as a hard constraint. If a constraint triggers only mild unacceptability and is subject to contextual variation and developmental optionality, then the constraint is soft. The classification can be verified by investigating the crosslinguistic behavior of the constraint; the type of a constraint (soft or hard) should remain the same across languages.

A model of gradience in grammar should account for the experimental properties of gradient linguistic structures identified by Sorace and Keller (2004) and summarized in this section. 
Table 1: Properties of hard and soft constraints, from Sorace \& Keller, 2004

\begin{tabular}{lll}
\hline & hard constraints & soft constraints \\
\hline universal & ranking effects & ranking effects \\
effects & cumulativity effects & cumulativity effects \\
& ganging up effects & ganging up effects \\
\hline type-specific & strong unacceptability & mild unacceptability \\
effects & no context effects & context effects \\
& no developmental optionality & developmental optionality \\
\hline crosslinguistic & constraints are hard & constraints are soft \\
effects & across languages & across languages \\
\hline
\end{tabular}

\section{Linear Optimality Theory}

Linear Optimality Theory as proposed by Keller (2000) is a model of gradience that makes predictions about the relative grammaticality of linguistic structures. It builds on core concepts from Optimality Theory, a framework that is attractive for this purpose as it is equipped with a notion of competition that makes it possible formalize the interaction of linguistic constraints. Furthermore, OT provides a notion of constraint ranking that makes it possible to account for the fact that constraints differ in strength, i.e., that some constraints are more important than others for the overall well-formedness of a given linguistic structure.

Although LOT borrows central concepts (such as constraint ranking and competition) from Optimality Theory, it differs in two crucial respects from existing OT-based accounts. Firstly, it relies on the assumption that constraint ranks are represented as sets of numeric weights, instead of as partial orders. Secondly, it assumes that the grammaticality of a given structure is proportional to the sum of the weights of the constraints it violates. This means that OT's notion of strict domination is replaced with an linear constraint combination scheme (hence the name Linear Optimality Theory).

Only a limited number of components of the OT architecture are affected by the switch to LOT. The changes concern only HEval, the function that evaluates the harmony of a candidate, and Rank, the ranking component. LOT does not affect assumptions concerning the input and the generation function Gen, the two components of an OT grammar that determine which structures compete with each other. Also the constraint component Con, i.e., formal apparatus for representing constraints and candidates is unaffected. The LOT approach is neutral in these respects, and compatible with the diverse assumptions put forward in the OT literature.

However, LOT's versions of HEval and Rank entail changes in the way the optimal candidate is computed, as well as requiring a new type of ranking argumentation, i.e., a method for establishing constraint ranks from a set of linguistic examples. It will be shown that this type of ranking argumentation is considerably simpler than the one classically assumed in OT. Also, well understood algorithms exist for automating this type of ranking 
argumentation.

\subsection{Violation profiles and harmony}

The most prominent pattern in the experimental data presented by Keller (2000) is the cumulativity of constraint violations, i.e., the fact that the degree of unacceptability of a structure increases with the number of constraint violations it incurs. Cumulativity was in evidence in data on extraction, binding, gapping, and word order. Keller (2000) shows that both soft and hard constraint violations are cumulative, and that the cumulativity effect extends from multiple violations of different constraints to multiple violations of the same constraint.

The other pervasive pattern in Keller's (2000) data is the ranking of constraints, i.e., the fact that constraint violations differ in the degree of unacceptability they cause. Constraint ranking was observed in data on extraction, binding, gapping, and word order. Again, the ranking of constraints seems to hold for both soft and hard constraints.

The LOT model of gradient grammaticality derives from these two fundamental findings about constraint cumulativity and constraint ranking. Two hypotheses implement these two results. The first hypothesis deals with constraint ranking:

\section{Ranking Hypothesis}

The ranking of linguistic constraints can be implemented by annotating each constraint with a numeric weight representing the reduction in acceptability caused by a violation of this constraint.

Note that this notion of constraint ranks as numeric weights is more general than the notion of ranks standardly assumed in Optimality Theory. Standard OT formulates constraint ranks as binary ordering statements of the form $C_{1} \gg C_{2}$, meaning that constraint $C_{1}$ is ranked higher than the constraint $C_{2}$. Such statements do not make any assumptions regarding how much higher the ranking of $C_{1}$ is compared to the ranking of $C_{2}$. Such information is only available once we adopt a numeric concept of constraint ranking.

In the remainder of this paper, we will adopt the following terminological convention. The term constraint weight will be used to refer to the numeric annotation that our model assigns to a constraint. The term constraint rank will be employed to refer to the relative weight of two constraints in our model: we say that a constraint outranks another constraint if it has a greater weight (see also Definition (9) below). This usage is justified by the fact that Standard OT ranks (i.e., constraint orderings) are a special case of ranks as defined in Linear Optimality Theory (this will be shown in Section 4.1).

Once numeric constraint weights have been postulated, the overall acceptability of a structure can be computed based on the weights of the constraints that the structure violates. We will assume that simple summation is sufficient to compute the degree of acceptability of a structure from the weights of the constraints that the structure violates. This will account straightforwardly for the cumulativity of constraint violations observed experimentally. Keller (2000) demonstrates that this approach achieves a good model fit on his experimental data. 
To account for the cumulativity of constraint weights, LOT formulates the Linearity Hypothesis in (2):

\section{Linearity Hypothesis}

The cumulativity of constraint violations can be implemented by assuming that the grammaticality of a structure is proportional to the weighted sum of the constraint violations it incurs, where the weights correspond to constraint ranks.

The hypotheses in (1) and (2) can be made explicitly by formulating a numeric model that relates constraints ranks and degree of grammaticality. This relies on the notion of a grammar signature, which specifies the constraint set and the associated weights for a grammar. (Note that this definition, and all subsequent ones, are independent of the formulation of the constraints proper; the LOT account is one of constraint interaction, not of actual linguistic constraints.)

\section{Grammar Signature}

A grammar signature is a tuple $\langle\mathbf{C}, w\rangle$ where $\mathbf{C}=\left\{C_{1}, C_{2}, \ldots, C_{n}\right\}$ is the constraint set, and $w\left(C_{i}\right)$ is a function that maps a constraint $C_{i} \in \mathbf{C}$ on its constraint weight $w_{i}$.

Relative to a grammar signature, a given candidate structure has a constraint violation profile as defined in (4). The violation specifies which constraints are violated by the structure and how often. This is a useful auxiliary notion that will be relied on in further definitions.

\section{Violation Profile}

Given a constraint set $\mathbf{C}=\left\{C_{1}, C_{2}, \ldots, C_{n}\right\}$, the violation profile of a candidate structure $S$ is the function $v\left(S, C_{i}\right)$ that maps $S$ on the number of violations of the constraint $C_{i} \in \mathbf{C}$ incurred by $S$.

Based on Definitions (3) and (4), the harmony of a structure can now be defined using a simple linear model:

\section{Harmony}

Let $\langle\mathbf{C}, w\rangle$ be a grammar signature. Then the harmony $H(S)$ of a candidate structure $S$ with a violation profile $v\left(S, C_{i}\right)$ is given in (6).

$$
H(S)=-\sum_{i} w\left(C_{i}\right) v\left(S, C_{i}\right)
$$

Equation (6) states that the harmony of a structure is the negation of the weighted sum of the constraint violations that the structure incurs. Intuitively, the harmony of a structure describes its degree of well-formedness relative to a given set of constraints. This notion corresponds closely to the definition of harmony assumed in Standard OT (Prince \& Smolensky, 1997, p. 1607) or Harmonic Grammar (Smolensky, Legendre, \& Miyata, 1992, p. 14). 
The assumption is that all constraint weights are positive, i.e., that $w_{i} \geq 0$ for all $i$. This means that only constraint violations influence the harmony of a structure. Constraint satisfactions will not change the harmony of the structure (including cases where a constraint is vacuously satisfied because it is not applicable). This assumption is in accordance with Keller's (2000) experimental results, in which only constraints violations were found to affect acceptability. This will discussed further in Section 4.2.

\subsection{Constraint competition and optimality}

Based on the definitions of violation profile and harmony proposed in the preceding section, LOT's notion of grammaticality can now be specified. Grammaticality is computed in terms of the relative harmony of two candidates in the same candidate set:

\section{Grammaticality}

Let $S_{1}$ and $S_{2}$ be candidate structures in the candidate set $\mathbf{R}$. Then $S_{1}$ is more grammatical than $S_{2}$ if $H\left(S_{1}\right)>H\left(S_{2}\right)$. This can be abbreviated as $S_{1}>S_{2}{ }^{1}$

A crucial difference between harmony and grammaticality follows from Definition (7). Harmony is an absolute notion that describes the overall well-formedness of a structure. Grammaticality, on the other hand, describes the relative ill-formedness of a structure compared with another structure. While it is possible to compare the harmony of two structures across candidates sets, the notion of grammaticality is only well-defined for two structures that belong to the same candidate set (i.e., share the same input). Therefore, Definition (7) (and the subsequent Definition (8)) provide a relative notion of well-formedness, in line with the optimality theoretic tradition.

Based on the definition of grammaticality in (7), we can define the optimal structure in a candidate set as the one with the highest relative grammaticality.

\section{Optimality}

A structure $S_{\text {opt }}$ is optimal in a candidate set $\mathbf{R}$ if $S_{\text {opt }}>S$ for every $S \in \mathbf{R}$.

A notion of constraint rank can readily be defined in LOT based on the relative weight of two constraints (see also the terminological note on ranks vs. weights in Section 3.1 above):

\section{Constraint Rank}

A constraint $C_{1}$ outranks a constraint $C_{2}$ if $w\left(C_{1}\right)>w\left(C_{2}\right)$. This can be abbreviated as $C_{1} \gg C_{2}$.

In what follows, we will illustrate the definitions for harmony, grammaticality, and optimality. Consider an example grammar with the constraints $C_{1}, C_{2}$, and $C_{3}$, and the constraints weights given in Table 2 . This table also specifies an example candidate set $S_{1}, \ldots, S_{4}$ and gives the violation profiles for these candidates. The harmony for each of these structures can be computed based on Definition (5).

\footnotetext{
${ }^{1}$ This usage differs from the standard OT usage, where harmonic ordering is denoted by " $\succ$ ", not " $>$ ".
} 
Table 2: Example violation profi le and harmony scores

\begin{tabular}{|c||c|c|c|c|}
\hline$w(C)$ & 4 & 3 & 1 & $H(S)$ \\
\hline$S_{1}$ & & $*$ & $*$ & -4 \\
\hline$S_{2}$ & & $*$ & $* *$ & -5 \\
\hline$S_{3}$ & & & $*$ & -1 \\
\hline$S_{4}$ & $*$ & & & -4 \\
\hline
\end{tabular}

The structure $S_{3}$ maximizes harmony, i.e., it incurs the least serious violation profile. It is therefore the optimal structure in the candidate set, i.e., it is more grammatical than all other candidate structures. The structures $S_{1}$ and $S_{4}$ are both less grammatical than $S_{3}$. $S_{1}$ and $S_{4}$ receive the same harmony scores, but for different reasons; $S_{4}$ because it incurs a high-ranked violation of $C_{1}, S_{1}$ because it accumulates violations of $C_{2}$ and $C_{3}$. The structure $S_{2}$ is less grammatical than $S_{1}$, as it incurs an additional violations of $C_{3}$. In total, we obtain the following grammaticality hierarchy: $S_{3}>\left\{S_{1}, S_{4}\right\}>S_{2}$.

This examples illustrates the three central properties of constraint interaction that were identified in Section 2. The first property is the ranking of constraints. $S_{3}$ incurs a violation of $C_{3}$, while $S_{4}$ incurs a violation of $C_{1}$. That $S_{3}$ is more grammatical than $S_{4}$ is accounted for by the fact that $C_{1}$ has a higher weight than $C_{3}$, i.e., the ranking $C_{1} \gg C_{3}$ holds. This is a situation that was observed many times in the experimental data presented by Keller (2000).

Furthermore, the example illustrates how the cumulativity of constraint violations is modeled. $S_{1}$ incurs single violations of $C_{2}$ and $C_{3}$. The structure $S_{2}$ also incurs a single violation of $C_{2}$, but a double violation of $C_{3}$. As a consequence, $S_{1}$ is more grammatical than $S_{2}$. Cumulativity effects such as these encountered frequently in Keller's (2000) experimental data.

Finally, Table 2 illustrates the ganging up of constraint violations. The structures $S_{1}$ and $S_{4}$ have different constraint profiles: $S_{4}$ violates the constraint $C_{1}$, while $S_{1}$ violates the two constraints $C_{2}$ and $C_{3}$, which are both lower ranked than $C_{1}$. However, $S_{1}$ and $S_{4}$ are equally grammatical because the two constraints $C_{2}$ and $C_{3}$ gang up against $C_{1}$, leading to the same harmony score in both structures. Again, this empirical patters is in evidence in Keller's (2000) experimental data.

Note that standard optimality theoretic evaluation of the candidate set in Table 2 leads to a different harmonic ordering: $S_{3}>S_{1}>S_{2}>S_{4}$. If we assume a naive extension of Standard OT, then this order corresponds to the grammaticality order of the candidates. The naive extension assumes the strict domination of constraints, and therefore fails to model ganging up effects. Under this approach, there is no possibility for a joint violation of $C_{2}$ and $C_{3}$ to be as serious as a single violation of $C_{1}$, due to the ranking $C_{1} \gg C_{2} \gg C_{3}$. Hence the naive extension of Standard OT fails to account for the ganging up effects that were observed experimentally. 


\subsection{Ranking argumentation and parameter estimation}

Optimality Theory employs so-called ranking arguments to establish constraint rankings from data. A ranking argument refers to a set of candidate structures with a certain constraint violation profile, and derives a constraint ranking from this profile. This can be illustrated by the following example: assume that two structures $S_{1}$ and $S_{2}$ have the same constraint profile, with the following exception: $S_{1}$ violates constraint $C_{1}$, but satisfies $C_{2}$. Structure $S_{2}$, on the other hand, violates constraint $C_{2}$, but satisfies $C_{1}$. If $S_{1}$ is acceptable but $S_{2}$ is unacceptable, then we can conclude that the ranking $C_{2} \gg C_{1}$ holds (see Prince \& Smolensky, 1993, 106).

In the general case, the fact that $S_{1}$ is acceptable but $S_{2}$ is unacceptable entails that each constraint violated by $S_{1}$ is outranked by at least one constraint violated by $S_{2}$. (See Hayes, 1997, for a more extensive discussion of the inference patterns involved in ranking argumentation in Standard OT.)

The LOT approach allows a form of ranking argumentation that relies on gradient acceptability data instead of the binary acceptability judgments used in Standard OT. A ranking argument in Linear Optimality Theory can be constructed based on the difference in acceptability between two structures in the same candidate set, using the following definition:

\section{Ranking Argument}

Let $S_{1}$ and $S_{2}$ be candidate structures in the candidate set $\mathbf{R}$ with the acceptability difference $\Delta H$. Then the equation in (11) holds.

$$
H\left(S_{1}\right)-H\left(S_{2}\right)=\Delta H
$$

This definition assumes that the difference in harmony between $S_{1}$ and $S_{2}$ is accounted for by $\Delta H$, the acceptability difference between the two structures. $\Delta H$ can be observed empirically, and measured, for instance, using magnitude estimation judgments (Sorace \& Keller, 2004). Drawing on the definition of harmony in (5), Equation (11) can be transformed to:

$$
\sum_{i} w\left(C_{i}\right)\left(v\left(S_{1}, C_{i}\right)-v\left(S_{2}, C_{i}\right)\right)=-\Delta H
$$

This assumes that $S_{1}$ and $S_{2}$ have the violation profiles $v\left(S_{1}\right)$ and $v\left(S_{2}\right)$ and are evaluated relative to the grammar signature $\langle\mathbf{C}, w\rangle$.

Typically, a single ranking argument is not enough to rank the constraints of a given grammar. Rather, we need to accumulate a sufficiently large set of ranking arguments, based on which we can then deduce the constraint hierarchy of the grammar. To obtain a maximally informative set of ranking arguments, we take all the candidate structures in a given candidate set and compute a ranking argument for each pair of candidates, using Definition (12). 
The number of ranking arguments that a set of $k$ candidates yields is given in (13); note that this is simply the number of all unordered pairs that can be generated from a set of $k$ elements.

$$
n=\frac{k^{2}-k}{2}
$$

Now we are faced with the task of computing the constraint weights of a grammar from a set of ranking arguments. This problem can be solved by regarding the set of ranking arguments as a system of linear equations. The solution for this system of equations will then provide a set of constraint weights for the grammar. This idea is best illustrated using an example. We consider the candidate set in Table 2 and determine all ranking arguments generated by this candidate set (here $w_{i}$ is used as a shorthand for $w\left(C_{i}\right)$, the weight of constraint $\left.C_{i}\right)$ :

$$
\begin{array}{lll}
S_{1}-S_{2}: & 0 w_{1}+1 w_{2}+1 w_{3}-0 w_{1}-1 w_{2}-2 w_{3}=-((-4)-(-5))= & -1 \\
S_{1}-S_{3}: & 0 w_{1}+1 w_{2}+1 w_{3}-0 w_{1}-0 w_{2}-1 w_{3}=-((-4)-(-1))=3 \\
S_{1}-S_{4}: & 0 w_{1}+1 w_{2}+1 w_{3}-1 w_{1}-0 w_{2}-0 w_{3}=-((-4)-(-4))=0 \\
S_{2}-S_{3}: & 0 w_{1}+1 w_{2}+2 w_{3}-0 w_{1}-0 w_{2}-1 w_{3}=-((-5)-(-1))=4 \\
S_{2}-S_{4}: & 0 w_{1}+1 w_{2}+2 w_{3}-1 w_{1}-0 w_{2}-0 w_{3}=-((-5)-(-4))=1 \\
S_{3}-S_{4}: & 0 w_{1}+0 w_{2}+1 w_{3}-1 w_{1}-0 w_{2}-0 w_{3}=-((-1)-(-4))= & -3
\end{array}
$$

This system of linear equations can be simplified to:

$$
\begin{array}{llr}
-w_{3} & = & -1 \\
w_{2} & = & 3 \\
w_{2}+w_{3}-w_{1} & = & 0 \\
w_{2}+w_{3} & = & 4 \\
w_{2}+2 w_{3}-w_{1} & = & 1 \\
w_{3}-w_{1} & = & -3
\end{array}
$$

We have therefore determined that $w_{2}=3$ and $w_{3}=1$. The value of $w_{1}$ can be easily be obtained from any of the remaining equations: $w_{1}=w_{2}+w_{3}=4$.

This example demonstrates how a system of linear equations that follows from a set of ranking arguments can be solved by hand. However, such a manual approach is not practical for large systems of equations as they occur in realistic ranking argumentation. Typically, we will be faced with a large set of ranking arguments, generated by a candidate set with many structures, or by several candidate sets.

There are a number of standard algorithms for solving systems of linear equations, which can be utilized for automatically determining the constraint weights from a set of ranking arguments. One example is Gaussian Elimination, an algorithm which delivers an exact solution of a system of linear equations (if there is one). If we are dealing with experimental data, then the set of ranking arguments derived from a given data set will often result in an inconsistent set of linear equations, which means that Gaussian Elimination is not applicable. In such a case, the algorithm of choice is Least Square Estimation (LSE), 
a method for solving a system of linear equations even if the system is inconsistent. This means that LSE enables us to estimate the constraint weights of an LOT grammar if there is no set of weights that satisfy all the ranking arguments exactly (in contrast to Gaussian elimination). LSE will find an approximate set of constraint weights that maximizes the fit with the experimentally determined acceptability scores. A more detailed explanation of LSE and its application to LOT is provided by Keller (2000).

\subsection{Evaluation of model fit and predictivity}

Once the parameters of an LOT model have been established using LSE, we need to evaluate how well the model accounts for a given set of ranking arguments. For Least Square Estimation, a standard metric of model $f i t$ is available in the form of the mean squared error, i.e., the mean of the difference between the model's predicted acceptability difference for a given ranking argument, and the actual acceptability difference found experimentally. The mean squared error of a model, $e_{\mu}$, can be defined as follows:

\section{Mean Squared Error of a Model}

Let $\Delta H_{i}$ be the acceptability difference for the item $i$ in the data set, and let $\Delta H_{i}^{\prime}$ be the acceptability difference predicted by the model for the item $i$. Then the mean squared error of the model is as given in (17), where $n$ is the number of items in the data set.

$$
e_{\mu}=\frac{1}{n} \sum_{i=1}^{n}\left(\Delta H_{i}-\Delta H_{i}^{\prime}\right)^{2}
$$

We also have to make sure that the model does not overfi $t$ the data, i.e., that it is not only able to account for the data that was used for parameter estimation, but can generalize to new data of the same type. In linguistic terms, this means that the model is predictive. The generalization ability of a model can be tested by applying it to unseen data, i.e., to data that it has not been used to estimate the model parameters. Again, the mean squared error can be used to quantify the model fit on the test data. If the model fit on the training data and on the test data are similar, then it can be concluded that the model is able to generalize.

Standard techniques from machine learning (Mitchell, 1997) and computational linguistics (Manning \& Schütze, 1999) can be used to carry out detailed studies of the behavior of a model on unseen data, as discussed in more detail by Keller and Asudeh (2002).

\section{Comparison with other Optimality Theoretic approaches}

\subsection{Standard Optimality Theory}

Linear Optimality Theory preserves key concepts of Standard Optimality Theory. This includes the fact that constraints are violable, even in an optimal structure. As in Standard OT, LOT avails itself of a notion of constraint ranking to resolve constraint conflicts; LOT's notion of ranking is quantified, i.e., richer than the one in Standard OT. The second 
core OT concept inherited by LOT is constraint competition. The optimality of a candidate cannot be determined in isolation, but only relative to other candidates it competes with. Furthermore, LOT uses ranking arguments in a similar way as Standard OT. Such ranking arguments work in a competitive fashion, i.e., based on the comparison of the relative grammaticality of two structure in the same candidate set. As in Standard OT, a comparison of structures across candidate sets is not well-defined; two structures only compete against each other if they share the same input.

The crucial difference between LOT and Standard OT is the fact that in LOT, constraint ranks are implemented as numeric weights and a straightforward linear constraint combination scheme is assumed. Standard Optimality Theory can then be regarded as a special case of LOT, where the constraint weights are chosen in an exponential fashion so as to achieve strict domination (see the Subset Theorem in (18)). The extension of Standard OT to LOT is crucial in accounting for the cumulativity of constraint violations. The linear constraint combination schema also greatly simplifies the task of determining a constraint hierarchy from a given data set. This problem simply reduces to solving a system of linear equations, a well-understood mathematical problem for which a set of standard algorithms exists (see Section 3.3).

Another advantage is that LOT naturally accounts for optionality, i.e., for cases where more than one candidate is optimal. Under the linearity hypothesis, this simply means that the two candidates have the same harmony score. Such a situation can arise if the two candidates have the same violation profile, or if they have different violation profiles, but the weighted sum of the violation is the same in both cases. No special mechanism for dealing with constraint ties are required in Linear OT. This is an advantage over Standard OT, where the modeling of optionality is less straightforward (see Asudeh, 2001, for a discussion).

An OT grammar can be formulated as a weighted grammar if the constraint weights are chosen in an exponential fashion, so that strict domination of constraints is assured. This observation is due to Prince and Smolensky (1993, p. 200) and also applies to Linear Optimality Theory. Therefore, the theorem in (18) holds (the reader is referred to Keller (2000) for a proof).

\author{
Subset Theorem \\ A Standard Optimality Theory grammar $G$ with the constraint set $\mathbf{C}=$ \\ $\left\{C_{1}, C_{2}, \ldots, C_{n}\right\}$ and the ranking $C_{n} \gg C_{n-1} \gg \ldots \gg C_{1}$ can be expressed as a \\ Linear Optimality Theory grammar $G^{\prime}$ with the signature $\langle\mathbf{C}, w\rangle$ and the weight \\ function $w\left(C_{i}\right)=b^{i}$, where $b-1$ is an upper bound for multiple constraint viola- \\ tions in $G$.
}

Note that the Subset Theorem holds only if there is an upper bound $b-1$ that limits the number of multiple constraint violations that the grammar $G$ allows. Such an upper bound exists if we assume that the number of violations incurred by each structure generated by $G$ is finite. This assumption seems to be generally true for OT grammars. 


\subsection{Harmonic Grammar}

Harmonic Grammar (Legendre, Miyata, \& Smolensky, 1990a, 1990b, 1991; Smolensky et al., 1992; Smolensky, Legendre, \& Miyata, 1993) is predecessor of OT that builds on the assumption that constraints are annotated with numeric weights (instead of just being rank-ordered as in Standard OT). Harmonic Grammar (HG) can be implemented in a hybrid connectionist-symbolic architecture and has been applied successfully to gradient data by Legendre et al. (1990a, 1990b). As Prince and Smolensky (1993, p. 200) point out, 'Optimality Theory [...] represents a very specialized kind of Harmonic Grammar, with exponential weighting of the constraints".

Linear Optimality Theory is similar to HG in that it assumes constraints that are annotated with numeric weights, and that the harmony of a structure is computed as the linear combination of the weights of the constraints it violates. There are, however, two differences between LOT and HG: (a) LOT only models constraint violations, while HG models both violations and satisfactions; and (b) LOT uses standard least square estimation to determine constraint weights, while $\mathrm{HG}$ requires more powerful training algorithms such as backpropagation. We will discuss each of these differences in turn.

LOT requires that all constraints weights have the same sign (only positive weights are allowed, see Section 3.1). This amounts to the claim that only constraint violations (but not constraint satisfactions) play a role in determining the grammaticality of a structure. In HG, in contrast, arbitrary constraint weights are possible, i.e., constraint satisfactions (as well as violations) can influence the harmony of a structure. This means that HG allows to define a grammar that contains a constraint $C$ with the weight $w$ and a constraint $C^{\prime}$ that is the negation of $C$ and has the weight $-w$. In such a grammar, both the violations and the satisfactions of $C$ influence the harmony of a structure.

The issue of positive weights has important repercussions for the relationship between Standard OT and LOT: Keller (2000) proves a Superset Theorem that states that an arbitrary LOT grammar can be simulated by a Standard OT grammar with stratified hierarchies. The proof crucially relies on the assumption that all constraint weights are of the same sign. Stratified hierarchies allow us to simulate the addition of constraint violations (they correspond to multiple violations in Standard OT), but they do not allow us to simulate the subtraction of constraint violations (which would be required by constraints that increase harmony). This means that the Superset Theorem does not hold for grammars that have both positive and negative constraints weights, as they are possible in Harmonic Grammar.

The second difference between HG and LOT concerns parameter estimation. An HG model can be implemented as a connectionist network, and the parameters of the model (the constraint weights) can be estimated using standard connectionist training algorithms. An example is provided by the HG model of unaccusativity/unergativity in French presented by Legendre et al. (1990a, 1990b) and Smolensky et al. (1992). This model is implemented as a multilayer perceptron and trained using the backpropagation algorithm (Rumelhart, Hinton, \& Williams, 1986).

It is well known that many connectionist models have an equivalent in conventional 
statistical techniques for function approximation. Multilayer perceptrons, for instance, correspond to a family of non-linear statistical models, as shown by Sarle (1994). (Which non-linear model a given perceptron corresponds to depends on its architecture, in particular the number and size of the hidden layers.) The parameters of a multilayer perceptron are typically estimated using backpropagation or similar training algorithms.

On the other hand, a single-layer perceptron (i.e., a perceptron without hidden layers) corresponds to multiple linear regression, a standard statistical technique for approximating a linear function of multiple variables. The parameters (of both a single-layer perceptron and a linear repression model) can be computed using least square estimation (Bishop, 1995). This technique can also be used for parameter estimation for LOT models (see Section 3.3). Note that LOT can be conceived of as a variant of multiple linear regression. The difference between LOT and conventional multiple linear regression is that parameter estimation is not carried directly on data to be accounted for (the acceptability judgments); rather, a preprocessing step is carried out on the judgment data to compute a set of ranking arguments, which then form the input for the regression.

To summarize, the crucial difference between HG and LOT is that HG is a non-linear function approximator, while LOT is a linear function approximator, i.e., a variant of linear regression. This means that a different set of parameter estimation algorithms is appropriate for HG and LOT, respectively.

\subsection{Probabilistic Optimality Theory}

Boersma and Hayes (2001) propose a probabilistic variant of Optimality Theory (POT) that is designed to account for gradience both in corpus frequencies and in acceptability judgments. POT stipulates a continuous scale of constraint strictness. Constraints are annotated with numerical strictness values; if a constraint $C_{1}$ has a higher strictness value that a constraint $C_{2}$, then $C_{1}$ outranks $C_{2}$. Boersma and Hayes (2001) assume probabilistic constraint evaluation, which means that at evaluation time, a small amount of random noise is added to the strictness value of a constraint. As a consequence, re-rankings of constraints are possible if the amount of noise added to the strictness values exceeds the distance between the constraints on the strictness scale.

For instance, assume that two constraints $C_{1}$ and $C_{2}$ are ranked $C_{1} \gg C_{2}$, selecting the structure $S_{1}$ as optimal for a given input. Under Boersma and Hayes's (2001) approach, a re-ranking of $C_{1}$ and $C_{2}$ can occur at evaluation time, resulting in the opposite ranking $C_{2} \gg$ $C_{1}$. This re-ranking might result in an alternative optimal candidate $S_{2}$. The probability of the re-ranking that makes $S_{2}$ optimal depends on the distance between $C_{1}$ and $C_{2}$ on the strictness scale (and on the amount of noise added to the strictness values). The re-ranking probability is assumed to predict the degree of grammaticality of $S_{2}$. The more probable the re-ranking $C_{2} \gg C_{1}$, the higher the degree of grammaticality of $S_{2}$; if the rankings $C_{1} \gg C_{2}$ and $C_{2} \gg C_{1}$ are equally probable, then $S_{1}$ and $S_{2}$ are equally grammatical.

The POT framework comes with its own learning theory in the form of the Gradual Learning Algorithm (Boersma, 1998, 2000; Boersma \& Hayes, 2001). This algorithm is a generalization of Tesar and Smolensky's (1998) Constraint Demotion Algorithm in that 
Table 3: Data that cannot be modeled in Probabilistic OT (hypothetical frequencies or acceptability scores), from Keller \& Asudeh, 2002

\begin{tabular}{|c|c|c|c|c|}
\hline /input/ & $C_{3}$ & $C_{1}$ & $C_{2}$ & Freq./Accept. \\
\hline$S_{1}$ & & $*$ & & 3 \\
\hline$S_{2}$ & & $*$ & $*$ & 2 \\
\hline$S_{3}$ & $*$ & & & 1 \\
\hline
\end{tabular}

Table 4: Data that cannot be modeled in Probabilistic OT (hypothetical frequencies or acceptability scores), from Keller \& Asudeh, 2002

\begin{tabular}{|c|c|c|c|}
\hline /input/ & $C_{1}$ & $C_{2}$ & Freq./Accept. \\
\hline$S_{1}$ & & $*$ & 4 \\
\hline$S_{2}$ & & $* *$ & 3 \\
\hline$S_{3}$ & & $* * *$ & 2 \\
\hline$S_{4}$ & $*$ & & 1 \\
\hline
\end{tabular}

it performs constraint promotion as well as demotion. The Gradual Learning Algorithm incrementally adjusts the strictness values of the constraints in the grammar to match the frequencies of the candidate structures in the training data. The fact that the algorithm relies on gradual changes makes it robust to noise, which is an attractive property from a language acquisition point of view.

There are, however, a number of problems with the POT approach. As Keller and Asudeh (2002) point out, POT cannot model cases of harmonic bounding, as a illustrated in Table 3: candidate $S_{2}$ is harmonically bound by candidate $S_{1}$, which means that there is no re-ranking of the constraints that would make $S_{2}$ optimal. As $S_{2}$ can never be optimal, its frequency or acceptability is predicated to be zero (i.e., no other candidate can be worse, even if it violates additional constraints). An example where this is clearly incorrect is $S_{3}$ in Table 3, which violates a higher ranked constraint and is less acceptable (or less frequent) than $S_{2}$.

A second problem with POT identified by Keller and Asudeh (2002) is cumulativity. This can be illustrated with respect to Table 4 : here, candidate $S_{1}$ violates constraint $C_{2}$ once and is more acceptable than $S_{2}$, which violates $C_{2}$ twice. $S_{2}$ in turn is more acceptable than $S_{3}$, which violates $C_{2}$ three times. A model based on constraint re-ranking cannot account for this, as a re-ranking of $C_{2}$ will not change the outcome of the competition between $S_{1}, S_{2}$, and $S_{3}$. Essentially, this is a special case of harmonic bounding involving only one constraint.

There is considerable evidence that configurations such as the ones illustrated in Tables 3 and 4 occur in real data. Keller (2000) reports acceptability judgment data for word 
Table 5: Data that cannot be modeled in $\mathrm{POT}^{\prime}$ (hypothetical frequencies or acceptability scores)

\begin{tabular}{|c|c|c|c|c|}
\hline /input/ & $C_{3}$ & $C_{1}$ & $C_{2}$ & Freq./Accept. \\
\hline$S_{1}$ & & $*$ & & 2 \\
\hline$S_{2}$ & & $*$ & $*$ & 1 \\
\hline$S_{3}$ & $*$ & & & 1 \\
\hline
\end{tabular}

order variation in German that instantiates both patterns. Guy and Boberg's (1997) frequency data for coronal stop deletion in English instantiates the cumulative pattern in Table 4. Jäger and Rosenbach (2004) show that cumulativity is instantiated in both frequency data and acceptability data on genitive formation in English. None of these data sets can be modeled by POT, and thus constitute serious counterexamples to this approach. In Linear Optimality Theory, on the other hand, such cases are completely unproblematic, due to the linear combination scheme assumed in this framework.

In a recent paper, Boersma (2004) acknowledges that cases of harmonic bounding and cumulativity as illustrated in Tables 3 and 4 pose a problem for POT. In response to this, he proposes a variant of POT, which we will call POT'. In $\mathrm{POT}^{\prime}$, the acceptability of a candidate $S$ is determined by carrying out a pairwise comparison between $S$ and each of the other candidates in the candidate set; the acceptability of $S$ then corresponds to the percentage of comparisons that $S$ wins. ${ }^{2}$ As an example, take the tableau in Table 3. Here, $S_{1}$ wins against $S_{2}$ and $S_{3}$, hence his acceptability value is $2 / 2=100 \%$. $S_{2}$ wins against $S_{3}$ but loses against $S_{1}$, so its acceptability is $1 / 2=50 \%$. $S_{3}$ loses against both candidates, and thus receives an acceptability value of $0 \%$.

In $\mathrm{POT}^{\prime}$, the relative grammaticality of a candidate corresponds to its optimality theoretic rank in the candidate set. This is not a new idea; in fact it is equivalent to the definition of relative grammaticality in terms of suboptimality, initially proposed by Keller (1997). The only difference is that in POT $^{\prime}$, suboptimality is determine based on a POT notion of harmony, instead of using the standard OT notion of harmony, as assumed by Keller (1997). However, there are a number of conceptual problems with this proposal (which carry over to $\mathrm{POT}^{\prime}$ ), discussed in detail by Müller (1999) and Keller (2000).

In addition to that, there are empirical problems with the $\mathrm{POT}^{\prime}$ approach. $\mathrm{POT}^{\prime}$ correctly predicts the relative acceptability of the example in Table 3 (as outlined above). However, other counterexamples can be constructed easily if we assume ganging up effects. In Table 5, the combined violation of $C_{1}$ and $C_{2}$ is as serious as the single violation of $C_{3}$, which means that the candidates $S_{2}$ and $S_{3}$ are equally grammatical. Such a situation can not be modeled in POT $^{\prime}$, as $S_{2}$ will win against $S_{3}$ (because $C_{3}$ outranks $C_{1}$ ), hence $S_{2}$ is predicted to be more grammatical than $S_{3}$. As discussed in Section 2, ganging up effects occur in experimental data, and thus pose a real problem for POT $^{\prime}$.

\footnotetext{
${ }^{2}$ More precisely, it is the POT probability of winning, averaged over all pairwise comparisons, but this difference is irrelevant here.
} 
In contrast to POT and $\mathrm{POT}^{\prime}$, LOT can model ganging up effects straightforwardly, as illustrated in Section 3.2. This is not surprising: the weights in LOT grammars are estimated so that they correspond in a linear fashion to the acceptability scores of the candidates in the training data. The strictness bands in POT (and POT') grammars, on the other hand, are estimated to match the frequencies of candidates in the training data; it is not obvious why such a model should correctly predict acceptability scores, given that it is trained on a different type of data.

\subsection{Maximum entropy models}

The problems with POT have led a number of authors to propose alternative ways of dealing with gradience in OT. Goldwater and Johnson (2003), Jäger (2004), and Jäger and Rosenbach (2004) propose a probabilistic variant of OT based on the machine learning framework of maximum entropy models, which is state of the art in computational linguistics (e.g., Abney, 1997; Berger, Della Pietra, \& Della Pietra, 1996). In Maximum Entropy OT (MOT) as formulated by Jäger (2004), the probability of a candidate structure (i.e., of an input-output pair $(o, i))$ is defined as:

$$
p_{\vec{r}}(o \mid i)=\frac{1}{Z_{\vec{r}}(i)} \exp \left(\sum_{j} r_{j} c_{j}(i, o)\right)
$$

Here, $r_{j}$ denotes the numeric rank of constraint $j$, while $\vec{r}$ denotes the ranking vector, i.e., the set of ranks of all constraints. The function $c_{j}(i, o)$ returns the number of violations of constraint $j$ incurred by input-output pair $(i, o) . Z_{\vec{r}}(i)$ is a normalization factor.

The model defined in (19) can be regarded as an extension of LOT as introduced in Section 3.1. It is standard practice in the literature on gradient grammaticality to model not raw acceptability scores, but log-transformed, normalized acceptability data (Keller, 2000). This can be made explicit by log-transforming the lefthand side of (6) (and dropping the minus and renaming the variable $i$ to $j$ ). The resulting formula is then equivalent to (20).

$$
H(S)=\exp \left(\sum_{j} w\left(C_{j}\right) v\left(S, C_{j}\right)\right)
$$

A comparison of (19) and (20) shows that the two models have a parallel structure: $w\left(C_{j}\right)=r_{j}$ and $v\left(S, C_{j}\right)=c_{j}(i, o)$ (the input-output structure of the candidates is implicit in (20)). Both models are instances of a more general family of models referred to as loglinear models. There is, however, a crucial difference between the MOT definition in (19) and the LOT definition in (20). Equation (20) does not include the normalization factor $Z_{\vec{r}}(i)$, which means that (20) does not express a valid probability distribution. The normalization factor is not trivial to compute, as it involves summing over all possible output forms $o$ (see Goldwater \& Johnson, 2003, and Jäger, 2004, for details). This is the reason why LOT can assumes a simple learning algorithm based on least square estimation, while MOT has to rely on learning algorithms for maximum entropy models, such as generalized iterative scaling, or improved iterative scaling (Berger et al., 1996). Another crucial difference between MOT and LOT (pointed out by Goldwater \& Johnson, 2003) is that MOT is 
designed to be trained on corpus data, while LOT is designed to be trained on acceptability judgment data.

\section{Conclusions}

This paper introduced Linear Optimality Theory (LOT) as model of gradient grammaticality. Although this model borrows central concepts (such as constraint ranking and competition) from Optimality Theory, it differs in two crucial respects from Standard OT. Firstly, LOT assumes that constraint ranks are represented as numeric weights (this feature is shared with Probabilistic OT and Maximum Entropy OT, see Sections 4.3 and 4.4). Secondly, LOT assumes that the grammaticality of a given structure is proportional to the sum of the weights of the constraints it violates, which means that OT's notion of strict domination is replaced with an linear constraint combination scheme (this feature is shared with Maximum Entropy OT, see Section 4.4).

We also outlined a learning algorithm for LOT (see Section 3.3). This algorithm takes as its input a grammar (i.e., a set of linguistic constraints) and a training set, based on which it estimates the weights of the constraints in the grammar. The training set is a collection of candidate structures, with the violation profile and the grammaticality score for each structure specified. Note that LOT is not intended as a model of human language acquisition: it cannot be assumed that the learner has access to training data that are annotated with acceptability scores. The sole purpose of the LOT learning algorithm is to perform parameter fitting for LOT grammars, i.e., to determine an optimal set of constraint weights for a given data set.

LOT is able to account for the properties of gradient structures discussed in Section 2. Constraint ranking is modeled by the fact that LOT annotates constraints with numeric weights representing the contribution of a constraint to the unacceptability of a structure. Cumulativity is modeled by the assumption that the degree of ungrammaticality of a structure is computed as the sum of the weights of the constraint the structure violates. Once ranking and cumulativity are assumed as part of the LOT model, other properties of gradient linguistic judgments follow without further stipulations. The ganging up effect is an obvious case: as constraint violations are additive, they can gang up. Also the distinction between soft and hard constraints does not have to stipulated in LOT; rather it emerges a consequence of LOT's assumptions about ranking and cumulativity and its learning algorithm. The LOT learning algorithm assigns high weights to constraints that cause serious unacceptability when violated, and low weights to constraints that cause only mild unacceptability. This means that all hard constraints will receive a similar constraint weight, and the same will be true for all soft constraints.

Another difference between soft and hard constraints is that soft, but not hard constraints can be subject to context effects. In LOT this makes the prediction that the weight of a soft constraint will vary with context, while the weight of a hard constraint will be stable across contexts. This prediction can be tested by applying the LOT learning algorithm to two training sets, containing acceptability scores for the same structures, but in two different contexts. The learning algorithm should compute the same weights for both 
training sets for hard constraints, while the weights for soft constraints can vary between the two sets. Keller (2000) presents modeling results that show that this prediction is born out for data on gapping and word order.

\section{References}

Abney, S. (1997). Stochastic attribute-value grammars. Computational Linguistics, 23(4), 597-618.

Asudeh, A. (2001). Linking, optionality, and ambiguity in Marathi. In P. Sells (Ed.), Formal and empirical issues in optimality-theoretic syntax. Stanford, CA: CSLI Publications.

Berger, A., Della Pietra, S., \& Della Pietra, V. (1996). A maximum entropy approach to natural language processing. Computational Linguistics, 22(1), 39-71.

Bishop, C. M. (1995). Neural networks for pattern recognition. Oxford: Oxford University Press.

Boersma, P. (1998). Functional phonology: Formalizing the interactions between articulatory and perceptual drives. The Hague: Holland Academic Graphics.

Boersma, P. (2000). Learning a grammar in functional phonology. In J. Dekkers, F. van der Leeuw, \& J. van de Weijer (Eds.), Optimality Theory: Phonology, syntax, and acquisition (pp. 465523). Oxford: Oxford Univeristy Press.

Boersma, P. (2004). A Stochastic OT account of paralinguistic task such as grammaticality and prototypicality judgments. In G. Fanselow, C. Féry, R. Vogel, \& M. Schlesewsky (Eds.), Gradience in grammar: Generative perspectives. Oxford: Oxford University Press.

Boersma, P., \& Hayes, B. (2001). Empirical tests of the gradual learning algorithm. Linguistic Inquiry, 32(1), 45-86.

Crocker, M., \& Keller, F. (2004). Probabilistic grammars as models of gradience in language processing. In G. Fanselow, C. Féry, R. Vogel, \& M. Schlesewsky (Eds.), Gradience in grammar: Generative perspectives. Oxford: Oxford University Press.

Fanselow, G., Féry, C., Vogel, R., \& Schlesewsky, M. (Eds.). (2004). Gradience in grammar: Generative perspectives. Oxford: Oxford University Press.

Goldwater, S., \& Johnson, M. (2003). Learning OT constraint rankings using a maximum entropy model. In J. Spenader, A. Eriksson, , \& Ö. Dahl (Eds.), Proceedings of the Stockholm workshop on variation within Optimality Theory (p. 111-120). Stockholm University.

Guy, G. R., \& Boberg, C. (1997). Inherent variability and the obligatory contour principle. Language Variation and Change, 9, 149-164.

Hayes, B. P. (1997). Four rules of inference for ranking argumentation. (Unpubl. ms., Department of Linguistics, University of California, Los Angeles)

Jäger, G. (2004). Maximum entropy models and Stochastic Optimality Theory. (Unpubl. ms., University of Potsdam) 
Jäger, G., \& Rosenbach, A. (2004). The winner takes it all - almost: Cumulativity in grammatical variation. (Unpubl. ms., University of Potsdam and University of Düsseldorf)

Keller, F. (1997). Extraction, gradedness, and optimality. In A. Dimitriadis, L. Siegel, C. SurekClark, \& A. Williams (Eds.), Proceedings of the 21st Annual Penn Linguistics Colloquium (pp. 169-186). Department of Linguistics, University of Pennsylvania.

Keller, F. (2000). Gradience in grammar: Experimental and computational aspects of degrees of grammaticality. Unpublished doctoral dissertation, University of Edinburgh.

Keller, F., \& Asudeh, A. (2002). Probabilistic learning algorithms and Optimality Theory. Linguistic Inquiry, 33(2), 225-244.

Legendre, G., Miyata, Y., \& Smolensky, P. (1990a). Harmonic grammar: A formal multi-level connectionist theory of linguistic well-formedness: An application. In Proceedings of the 12th Annual Conference of the Cognitive Science Society (pp. 884-891). Mahwah, NJ: Lawrence Erlbaum Associates.

Legendre, G., Miyata, Y., \& Smolensky, P. (1990b). Harmonic grammar: A formal multi-level connectionist theory of linguistic well-formedness: Theoretical foundations. In Proceedings of the 12th Annual Conference of the Cognitive Science Society (pp. 388-395). Mahwah, NJ: Lawrence Erlbaum Associates.

Legendre, G., Miyata, Y., \& Smolensky, P. (1991). Unifying syntactic and semantic approaches to unaccusativity: A connectionist approach. In Proceedings of the 17th Annual Meeting of the Berkeley Linguistics Society (pp. 388-395). Berkeley.

Manning, C. D., \& Schütze, H. (1999). Foundations of statistical natural language processing. Cambridge, MA: MIT Press.

Mitchell, T. M. (1997). Machine learning. New York: McGraw-Hill.

Müller, G. (1999). Optimality, markedness, and word order in German. Linguistics, 37(5), 777-818.

Prince, A., \& Smolensky, P. (1993). Optimality Theory: Constraint interaction in generative grammar (Technical Report No. 2). Center for Cognitive Science, Rutgers University.

Prince, A., \& Smolensky, P. (1997). Optimality: From neural networks to universal grammar. Science, 275, 1604-1610.

Proceedings of the 12th Annual Conference of the Cognitive Science Society. (1990). Mahwah, NJ: Lawrence Erlbaum Associates.

Rumelhart, D. E., Hinton, G. E., \& Williams, R. J. (1986). Learning internal representations by error propagation. In Parallel distributed processing: Explorations in the microstructure of cognition (Vol. 1: Foundations, pp. 318-362). Cambridge, MA: MIT Press.

Sarle, W. S. (1994). Neural networks and statistical models. In Proceedings of the 19th annual SAS users group international conference (pp. 1538-1550). Cary, NC. 
Smolensky, P., Legendre, G., \& Miyata, Y. (1992). Principles for an integrated connectionist/symbolic theory of higher cognition (Report No. CU-CS-600-92). Computer Science Department, University of Colorado at Boulder.

Smolensky, P., Legendre, G., \& Miyata, Y. (1993). Integrating connectionist and symbolic computation for the theory of language. Current Science, 64, 381-391.

Sorace, A., \& Keller, F. (2004). Gradience in linguistic data. Lingua, to appear.

Tesar, B., \& Smolensky, P. (1998). Learnability in Optimality Theory. Linguistic Inquiry, 29(2), 229-268. 